\title{
Malaria Outbreak Investigation in Argoba District, South Wello Zone, Northeast Ethiopia, 2016: A case control study
}

Getachew Yismaw workie

South Wollo Zonal Health Department

Getayeneh Antehunegn Tesema

University of Gondar

Ayenew Molla Lakew ( $\square$ mayenew15@gmail.com )

Institute of Public Health, College of Medicine and Health sciences, University of Gondar, Ethiopia https://orcid.org/0000-0003-3648-9891

Temesgen Yihunie Akalu

University of Epidemiology and Biostatistics

Research article

Keywords: malaria, outbreak, Argoba district

Posted Date: July 25th, 2019

DOI: https://doi.org/10.21203/rs.2.11960/v1

License: (9) This work is licensed under a Creative Commons Attribution 4.0 International License.

Read Full License 


\section{Abstract}

Background Malaria remains a major global public health concern and approximately half of the world's population being at risk of infection. It is one of the common epidemic prone diseases and can result in continuous epidemics and end up with serious public health emergencies. Therefore, this study aimed at identifying determinants of malaria outbreak in Argoba district. Methods Community based case-control study was employed with a 1:1 case to control ratio at Argoba district from 21 June to 7 July, 2016. A total of 150 individuals (75 cases and 75 controls) were included in the study. Cases were taken from treatment registration book and neighborhoods to the cases were taken as control groups. Structured questionnaire was used as data collection tool. Data was entered using Epi-info version 7 statistical software and exported to STATA version 14 for analysis. A multivariable logistic regression analysis was used to identify determinants of the outbreak. The magnitude of the outbreak was described by place, person, and time. Adjusted Odds Ratios (AOR) with the corresponding 95\% Confidence Interval (Cl) was used to show the strength of associations and variables with P-values of $<0.05$ were considered statistically significant. Results The predominant Plasmodium species detected was P.falciparum, which is followed by P.vivax. The overall attack rate was $1.8 \%$. Travel history to malarias site (AOR=14.6: $95 \% \mathrm{Cl}$ : $2.8,77.7$ ) and presence of mosquito breeding site around the home (less than 1000 meter) (AOR=13.3: $95 \% \mathrm{Cl}: 3.1,58.0)$ were associated with the occurrence of malaria outbreak. However, using insecticide treated bed nets $(\mathrm{AOR}=0.004 ; 95 \% \mathrm{Cl}: 0.001,0.06)$ and chemical indoor residual spray $(\mathrm{AOR}=0.02 ; 95 \% \mathrm{Cl}$ : $0.01,0.09$ ) were found to be protective factors. Conclusions malaria outbreak was affected by distance from the breeding site and traveling history of malaria endemic areas. In contrast, bed net utilization and house hold spray with DDT were preventive factors of the outbreak. It is recommended to use bed net utilization and house hold spraying in the district.

\section{Background}

Malaria is an acute febrile illness caused by a parasite known as plasmodium species and transmitted by the bites of infected mosquitoes (1). Four main species of malaria infect humans are: Plasmodium falciparum, Plasmodium vivax, Plasmodium malaria, and Plasmodium ovale (2). Plasmodium falciparum is the most dominant highly virulent species and is responsible for almost all of the 1.7-2.5 million deaths worldwide $(3,4)$. It is one of the world's most common and serious tropical diseases $(5)$.

Malaria is a leading public health problem in Ethiopia where an estimated 55.7 million people (68\% of the population) are at risk and three-quarter of the total land mass is regarded as malarias (6-8). The disease causes an average of 5 million cases and 70,000 deaths each year and accounts for $17 \%$ of outpatient visits to health institutions in Ethiopia (9). Ethiopia is among the few countries with unstable malaria transmission (10). Malaria is mostly an endemic disease, it may also occur as outbreaks, most of the malaria transmissions occur between September and December, after the main rainy season and from June to August (10). Certain areas, largely in the western and eastern parts of the country, experience a second "minor" malaria transmission period from April to May, following a short rainy season from February to March (11). Ethiopia is also one of the most malaria epidemic-prone countries in Africa. Since 
2005, Ethiopia has scaled-up one of the largest and most ambitious malaria control programs in Africa, designed to support the country's Health Sector Development Plan (HSDP), the national strategic plan (NSP) and the national child survival strategy, in order to reduce under-five mortality rates by two thirds by 2015 (12).

Possible precipitating factors of Malaria epidemics are: - abnormal weather conditions / rain fall, increase of vector capacity, deterioration of vector control operations, immigration of non- endemic into an endemic area, resistance to anti malaria drugs and constructions (like dams, mining ) (12-16).

While Ethiopia aims to achieve malaria elimination in selected geographical areas with historically low malaria transmission by 2015 , outbreaks occurred in some areas of the country (17). Therefore, this study was aimed at investigating and identifying determinants of malaria out-break in Argoba district, Northeast Ethiopia.

\section{Methods}

\section{Study design and setting}

Unmatched 1:1 case control study was conducted in Argoba district, which is one of the districts in south Wollo Zone, Amhara region, from June 21 to July 7, 2016. The district is divided in to 7 kebele (the smallest administrative division) with a total population of 39,873. The altitudes of the district were $300-$ 2100 Meter above sea level ( $90 \%$ Kola, 5\% Woina Dega and 5\% Dega) with annual rain fall of 500-750 $\mathrm{mmHg}$ and the temperature of 29-39.50 degree centigrade. It has 2 health centers and 7 health posts with 12 health extension workers which are currently on service (Fig 1).

\section{Population and sample}

All individuals in the district were considered as a source population. The required sample size was determined by using Epi-Info version 7 Statistical software using power approach formula, considering the $95 \%$ confidence level and $80 \%$ power (15). However, there were a total of 75 cases during the study period. 75 case and 75 controls were included in the study. Cases were taken from treatment registration book/line listing and neighborhoods to the cases were taken as control, lottery method was applied to select individual participants in the selected household for interview.

\section{Data collection and variable of the study}

Data was collected by using structured questionnaire by two health extension workers and two laboratory technicians under the supervision of the principal investigator and two BSC nurses. The questionnaire used in the study was developed for this particular study, and it was developed by reviewing literatures. One day training was given for data collectors and supervisors to maintain the data quality. Review of weekly Integrated Disease surveillance and Response (IDSR) report at different level (district health office 
and Health facilities), visiting the affected kebele, house to house visits were made to search for additional cases. Both Rapid Diagnostic Test (RDT) and Microscopic laboratory diagnosis test were also performed. A line listing of the cases were done.

Health extension workers used RDTS to identify confirmed malaria cases at health post and community level during outbreak investigation. Laboratory technicians used microscope to detect malaria parasites at Medina Health Center of Argoba district. Additionally, RDTS were also used in this health center during the interruption of electric power.

Data was also collected about the mosquito breeding sites from the district health office and health facilities. Cases/patients and controls were interviewed about presence of mosquito breeding sites around/near to their home. Similarly, observation on these potential mosquito breeding sites was made by health extension workers.

The dependent variable, presence of malaria (cases), was defined as an acute febrile illness with a peripheral blood smear positive for malaria parasite or a positive rapid antigen test by the RDTs. Whereas, controls were defined as when there were no signs and symptoms of acute febrile illness one month prior to the data collection. The independent variables were socio-demographic and economic factors (age, sex, occupation, educational status, and religion), behavioral factors (ITN utilization,

Dichlorodiphenyltrichloroethane (DDT) sprays of households, sleeping area during night) and human and environmental factors.

\section{Confirm the diagnosis and verify the existence of the outbreak}

Previous five years malaria data was reviewed from Argoba district health office and health facility to set epidemic threshold level. However, due to incompleteness of the previous five years data, last year's $(2015 / 16)$ weekly malaria cases report was used to set epidemic threshold level by comparing weekly data with similar week of 2016/17, an epidemic curve was constructed. An increase above the weekly threshold was observed, it implies an epidemic has been occurred. Blood sample were taken from 20 fever cases and tested by microscopy.

\section{Receiving rumor and prepared for field}

An early warning alarm was reported on June 19, 2016 from Argoba district. Following the request from the district health office and analysis of routine surveillance data, Zonal PHEM case team decided to investigate/ confirm the outbreak and to take intervention. Checklist/semi-structured questioner was prepared and necessary logistics were collected from zonal health department. The team were arrived to 
district on June 20, 2016 and discussed with district health office and RRT about the reported malaria outbreak rumor.

\section{Laboratory methods}

Laboratory technicians used microscope to detect malaria parasites at Medina Health Center of Argoba district. Additionally, RDTS were also used in this health center during the interruption of electric power. Health extension workers used RDTS to identify confirmed malaria cases at health post and Community level during outbreak investigation.

\section{Environmental assessment}

Data was collected about the mosquito breeding sites from the district health office and health facilities. Cases/patients and controls were interviewed about presence of mosquito breeding sites in their compound and near to home. Similarly, observation of these potential mosquito breeding sites and presence of anopheles larvae in stagnant water was conducted.

\section{Data processing and analysis}

Data was entered using Epi-info version 7 statistical software and exported to Statistical Package for Social Science (SPSS) version 20 for analysis. Descriptive and summary statistics were conducted to describe study variables. The Magnitude of outbreak was described by person, place and time. Variables with $p$-value less than 0.05 in the multivariable logistic regression analysis were considered as statistically significant. Odds ratio with $95 \%$ confidence level was used to measure the presence and strength of the association between the malaria case and contributing factors. Hosmer and Lemeshow goodness of fit test was conducted to test the model fitness.

The ethical approval was waived by the institutional review board of the University of Gondar. Formal letter of cooperation was written from Argoba district health office.

\section{Results}

\section{Magnitude of the outbreak by person}

During the outbreak investigation period, about 75 malaria cases were identified. The overall malaria attack rate was $1.8 \%$ and there was no death during the time of epidemic (Table 1). 
Table 1: Malaria cases and deaths by age and sex from week 25 to week 28, Argoba district, South Wollo, Northeast Ethiopia, 2016

\begin{tabular}{llll} 
Characteristics & Population & Cases & AR (\%) \\
\hline Age & & & \\
$<5$ year & 672 & 5 & $0.74 \%$ \\
$\geq 5$ year & 4303 & 70 & $1.63 \%$ \\
\hline Sex & & & \\
Male & 2569 & 51 & $1.99 \%$ \\
Female & 2406 & 24 & $1.00 \%$
\end{tabular}

More than two-third (68\%) of cases was males and majority (57.3\%) of them were in the age group 15-44. About $95 \%$ of case and $93 \%$ of controls were unemployed and $50.7 \%$ of cases and $54.7 \%$ of controls were unable to read and write (Table 2).

Table 2: Socio-demographic characteristics of study participants $(n=150)$ of malaria outbreak, Argoba district, South Wollo, Northeast Ethiopia, 2016 


\begin{tabular}{lll} 
Characteristics & Cases (\%) & Controls (\%) \\
\hline Gender & & \\
\hline Male & $51(68)$ & $49(65)$ \\
\hline Female & $24(32)$ & $26(35)$ \\
\hline Age group (years) & & \\
\hline$<5$ & $5(6.7)$ & $0(0)$ \\
\hline $5-14$ & $22(29.3)$ & $3(4)$ \\
\hline $15-44$ & $43(57.3)$ & $62(82.7)$ \\
\hline$>45$ & $5(6.7)$ & $10(13.3)$ \\
\hline Ethnicity & & \\
\hline Argoba & $72(96)$ & $73(97.3)$ \\
\hline Amhara & $3(4)$ & $2(2.7)$ \\
\hline Occupation & & \\
\hline Unemployed & $71(94.7)$ & $70(93.3)$ \\
\hline Governmental employee & $4(5.3)$ & $5(6.7)$ \\
\hline Educational status & & \\
\hline Unable to read and write & $38(50.7)$ & $41(54.7)$ \\
\hline Primary(1-8) & $33(44)$ & $27(36.0)$ \\
\hline Secondary and above & $4(5.3)$ & $7(9.3)$ \\
\hline
\end{tabular}

The baseline incidence rate for malaria prior to the outbreak was $0.8 / 1000$ population (at the starting of week 25) and the incidence at peak of the outbreak was 7.02/1000 population (week 26) then decreased to $2.21 / 1000$ population in the last week of the outbreak (week-28).

\section{Magnitude of the outbreak by time}

The baseline incidence rate for malaria prior to the outbreak was $0.8 / 1000$ population (at the starting of week 25) and the incidence at peak of the outbreak was 7.02/1000 population (week 26) then decreased to $2.21 / 1000$ population in the last week of the outbreak (week-28). The analyzed data indicated that 
alert threshold had been reached in week 24 ( 6 cases reported for a threshold of 6) and start crossing in week 25 (16 cases reported for a threshold of 8 stay above the threshold level up to week 28 (Fig 2).

\section{Magnitude of the outbreak by place}

Among seven kebeles in the district, the Gobera kebele was hosted malaria outbreak (Fig 3).

\section{Laboratory}

From the start of week 25 to week 28, a total of 223 malaria blood smear tests (both thick and thin films) were done by Microscopy and/or RDTs for suspected malaria cases as part of their routine work with a $100 \times$ oil immersion microscope in Medina Health Center, including the catchment health posts (Gobera HP). Out of total tested, 75 were positive for malaria (slide positivity rate: $39 \%)$, of which $82(96.0 \%)$ were P. falciparum. All positive slides and $10 \%$ of the negative slides were sent to the zonal level.

\section{Environmental assessment}

Breeding site of mosquitoes in the kebele was assessed by the integration of district health office and medina health center. The potential breeding sites were ponds, slow moving rivers, and streams within five kilometer radius of the affected communities. Larvae of anopheles mosquito were found in one river (of intermittently stagnant water bodies) and larvae were collected to show and educate health professionals and health extension workers working in the district (Table 3).

Table 3: Environmental and other related characteristics of study participants $(n=150)$ of malaria outbreak, Argoba district, South Wollo, Northeast Ethiopia, 2016 


\section{Characteristics}

Travel History

\begin{tabular}{lll}
\hline No & $43(57.3)$ & $64(85.3)$ \\
\hline Yes & $32(42.7)$ & $11(14.7)$ \\
\hline
\end{tabular}

Possession of bed net

\begin{tabular}{lll}
\hline No & $12(16)$ & $1(1.3)$ \\
\hline Yes & $63(84)$ & $74(98.7)$ \\
\hline
\end{tabular}

Utilization of Bed Net

\begin{tabular}{lll} 
No & $47(62.67)$ & $0(0.0)$ \\
\hline Yes & $28(37.33)$ & $75(100.0)$ \\
\hline
\end{tabular}

Spray status

\begin{tabular}{lll} 
No & $49(65.3)$ & $11(14.7)$ \\
Yes & $26(34.7)$ & $64(85.3)$ \\
\hline
\end{tabular}

\section{Presence of mosquito breeding site}

$\begin{array}{lll}\text { No } & 14(18.7) & 47(62.7) \\ \text { Yes } & 61(81.3) & 28(37.3)\end{array}$

\section{Sleeping preference}

Inside house

48(64.0) 70(93.3)

Outside house

27(36.0) 5(6.7)

\section{Factors associated with malaria outbreaks}

In multivariable logistic regression analysis age, travel history, chemical indoor residual spray, and presence of breading site near to home were found to have statistically significant association with malaria outbreak. The odds of having malaria was about 11 times higher among individuals having travel history to malarias area in the past two weeks than those did not ( $A O R=10.95,95 \% \mathrm{Cl}: 2.9,41.26)$. Individuals who were living in area with mosquito breeding site around their home were 17 times (AOR=17.01, 95\% Cl: 4.5, 64.31) more likely to had malaria as compared to others. The odds of having 
malaria among individuals who live in house with chemical indoor residual spray was decreased by 97.7 $\%$ than those do not. The odds of having malaria among 15 to 44 years decreased by $95.9 \%$ compared to age less than 15 years (Table 4).

Table 4: multiple Logistic regression analysis of independent factors related to malaria outbreak, Argoba district, South Wollo, Northeast Ethiopia, 2016

\begin{tabular}{llll} 
Characteristics & Cases (\%) & Controls (\%) & AOR (95\% Cl) \\
\hline Gender & & & \\
\hline Male & $51(68)$ & $49(65)$ & 1 \\
\hline Female & $24(32)$ & $26(35)$ & $0.44(0.12,1.58$ \\
\hline Age group (years) & & & \\
\hline $5-14$ & $27(36)$ & $3(4)$ & 1 \\
\hline $15-44$ & $43(57.3)$ & $62(82.7)$ & $0.04(0.01,0.24)$ \\
\hline$\geq 45$ & $5(6.7)$ & $10(13.3)$ & $0.11(0.013,0.86)$ \\
\hline
\end{tabular}

\section{Educational status}

\begin{tabular}{llll}
\hline Unable to read and write & $38(50.7)$ & $41(54.7)$ & 1 \\
\hline Primary(1-8) & $33(44)$ & $27(36.0)$ & $1.37(0.43,4.34)$ \\
\hline Secondary and above & $4(5.3)$ & $7(9.3)$ & $0.27(.036,1.97)$ \\
\hline Travel History & & & \\
\hline No & $43(57.3)$ & $64(85.3)$ & 1 \\
\hline Yes & $32(42.7)$ & $11(14.7)$ & $10.95(2.90,41.26)$
\end{tabular}

\section{Spray status}

\begin{tabular}{llll}
\hline No & $49(65.3)$ & $11(14.7)$ & 1.00 \\
\hline Yes & $26(34.7)$ & $64(85.3)$ & $0.02(0.01,0.09)$ \\
\hline Presence of mosquito breeding site & & & \\
\hline No & $14(18.7)$ & $47(62.7)$ & 1.00 \\
\hline Yes & $61(81.3)$ & $28(37.3)$ & $17.01(4.5,64.31)$
\end{tabular}

\section{Discussion}

Outbreaks of malaria are often complex, multi-factorial and may have natural and human made determinants (18). Findings from Argoba district showed that having travel history to malaria endemic 
area, living in house with chemical indoor residual spray, younger age and presence of mosquito breeding site around their home were significantly associated with outbreaks of malaria. A national malaria guideline in Ethiopia states that an epidemic starts when the number of cases in a given week is higher than the threshold number (either the third quartile or double the number in previous year) (12). Likewise, in this study it was crossed from weeks 25 to 28 . An increase above the weekly threshold were observed, it implies that there was an epidemic.

The predominant Plasmodium species detected was P. falciparum, followed by P. vivax. This was in agreement with other previous studies done in rural area of Ethiopia (19) and Kersa district (20).

In all age groups, malaria was reported in the study area. However, significantly affected age groups were 5-14 followed by 15-29 years old. This was in line with a study done in Metema district (21). This could be associated with high temperature in the area which is suitable for mosquito development rate, biting rate, and survival of the parasite within the mosquito. Besides, in these areas farming was the most dominant occupation and children may have minimal care. This may expose them to the bite of mosquitoes.

The study revealed that using bed net every night and spraying of houses with chemicals were associated with lower odds of malaria infection. Which is In line with the study done in Oromia Region (22). This might be due to the barrier effect of bed net utilization and/or it might be due to spraying of houses with chemical decrease the density of mosquitoes. The presence of mosquito breeding site around home was increasing the odds of malaria outbreak. Similar analytic approach in Sri Lanka and India indicated that people living closer to vector breeding sites were at higher odds of malaria than those living far away $(13,15)$. This might be due to increment of mosquito density because of the breeding site and /or risk for mosquitoes bite. The limitation of this study was self reporting on the measurement of ITN utilization which may cause misclassification bias.

\section{Conclusions}

Findings of this study showed that the dominant malaria species was P. falciparum. Mosquito breeding site closer to living house and travel history to malarias site were statistically significant predictors of malaria outbreak. However, spraying of house and using mosquito bed net were associated with lower odds of malaria. Therefore, health planners and administrators need to provide ITN for the community and the daily laborers that mobilized around Argoba about the control and prevention of malaria.

\section{Public health Intervention}

Test with RDT/microscopy and early case management were made. More than 1000 ITNs were distributed to the community. Environmental management (like filed, drain, clear) was made in more than 240 square meter area in selected Anopheles mosquitoes breeding site. A total of 289 (205 male and 84 female) population were participated in Environmental management. In addition, health education was 
given to the affected community member about the cause, transmission and prevention and control of malaria.

\section{List Of Abbreviations}

AOR: Adjusted Odds Ratio, BSC: Bachelor of Science, Cl: Confidence Interval, DDT:

Dichlorodiphenyltrichloroethane, IRS: Indoor Residual Spray, LLINS: Long Lasting Insecticidal Nets, ITN: Insecticide treated bed nets, and SPSS: Statistical Package for Social Science.

\section{Declarations}

\section{Ethics approval and consent to participate}

Official permission letter was obtained from Argoba district health office. Verbal informed consent was obtained from each study participant whose age was 17 and above. For the study participants whose age was under 17, verbal assent was obtained from each study participant after verbal consent was obtained from their parents or guardians. Confidentiality of the information was maintained properly and names and other personal identifiers were not recorded.

\section{Consent for publication}

Not applicable

\section{Availability of data and material}

Data will be available upon request from the corresponding author

\section{Competing interests}

Authors declare that they have no conflict of interest

\section{Funding}

No funding was obtained for this study.

\section{Authors' contributions}

GYW, GAT, AML and TYA were responsible for conceptualizing the research question, data curator, developing the analytic plan, analyzing the data and design and took the lead in writing the manuscript. All authors have read and approved the manuscript.

\section{Acknowledgments}


The authors forward their gratitude to Argoba district health facilities. We also grateful to our data collectors without them this study was not materialized.

\section{References}

1. Eckhoff PA. A malaria transmission-directed model of mosquito life cycle and ecology. Malaria journal. 2011;10(1):303.

2. Pérez-Tris J, Hasselquist D, Hellgren 0 , Krizanauskiene A, Waldenström J, Bensch S. What are malaria parasites? Trends in parasitology. 2005;21(5):209-11.

3. Idro R, Jenkins NE, Newton CR. Pathogenesis, clinical features, and neurological outcome of cerebral malaria. The Lancet Neurology. 2005;4(12):827-40.

4. Murphy SC, Breman JG. Gaps in the childhood malaria burden in Africa: cerebral malaria, neurological sequelae, anemia, respiratory distress, hypoglycemia, and complications of pregnancy. The American journal of tropical medicine and hygiene. 2001;64(1_suppl):57-67.

5. O'Meara WP, Mangeni JN, Steketee R, Greenwood B. Changes in the burden of malaria in subSaharan Africa. The Lancet infectious diseases. 2010;10(8):545-55.

6. Organization WH. Guidelines for the treatment of malaria: World Health Organization; 2015.

7. MoH F. Ethiopia National Malaria Indicator Survey 2015. 2017.

8. Health FMo. National five year strategic plan for malaria prevention and control in Ethiopia: 20062010. 2006.

9. Gabriel S, James V, editors. Developing malaria earky warning system for Ethiopia. 25th Annual ESRI International User Conference; 2005: National Center for EROS.

10. Alelign A, Dejene T. Current status of malaria in Ethiopia: evaluation of the burden, factors for transmission and prevention methods. Acta Parasitol Glob. 2016;7:1-6.

11. Centre EHaNRIHEM. Public Health Emergency Management guidelines for Ethiopia. Addis Ababa Ethiopia: February 2012.

12. Health FMo. National Malaria Guidelines Third Edition. Addis Abeba Ethiopia: minister of health, January 201.

13. Van Der Hoek W, Konradsen F, Amerasinghe PH, Perera D, Piyaratne M, Amerasinghe FP. Towards a risk map of malaria for Sri Lanka: the importance of house location relative to vector breeding sites. International Journal of Epidemiology. 2003;32(2):280-5.

14. Yeshiwondim AK, Gopal S, Hailemariam AT, Dengela DO, Patel HP. Spatial analysis of malaria incidence at the village level in areas with unstable transmission in Ethiopia. International Journal of Health Geographics. 2009;8(1):5.

15. Sharma PK, Ramanchandran R, Hutin YJ, Sharma R, Gupte MD. A malaria outbreak in Naxalbari, Darjeeling district, West Bengal, India, 2005: weaknesses in disease control, important risk factors. Malaria journal. 2009;8(1):288. 
16. The Carter Center A, Georgia. 4th ANNUAL MALARIA CONTROL PROGRAM REVIEWEthiopia and Nigeria. Atlanta, Georgia: carter center, March 8, 2013.

17. Abeku TA, De Vlas SJ, Borsboom G, Teklehaimanot A, Kebede A, Olana D, et al. Forecasting malaria incidence from historical morbidity patterns in epidemic-prone areas of Ethiopia: a simple seasonal adjustment method performs best. Tropical medicine \& international health. 2002;7(10):851-7.

18. Alonso PL, Tanner M. Public health challenges and prospects for malaria control and elimination. Nature medicine. 2013;19(2):150.

19. Manuel Ramos J, Reyes F, Tesfamariam A. Change in epidemiology of malaria infections in a rural area in Ethiopia. Journal of travel medicine. 2005;12(3):155-6.

20. Karunamoorthi K, Bekele M. Prevalence of malaria from peripheral blood smears examination: a 1year retrospective study from the Serbo Health Center, Kersa Woreda, Ethiopia. Journal of infection and Public Health. 2009;2(4):171-6.

21. Ferede G, Worku A, Getaneh A, Ahmed A, Haile T, Abdu Y, et al. Prevalence of malaria from blood smears examination: a seven-year retrospective study from Metema Hospital, Northwest Ethiopia. Malaria research and treatment. 2013;2013.

22. Deressa W, Chibsa S, Olana D. The distribution and magnitude of malarian in Oromia, Ethiopia. Ethiopian Journal of Health Development. 2004;18(3):163-70.

\section{Additional Files}

Fig 1: Map of Argoba district, 2016. Shows the location of Argoba district which is located at the East of the zone, south wollo Zone.

Fig 2: Malaria cases by weeks in Gobera, Argoba district, 2016. Illustrates distribution of malaria by time in the district. The magnitude of the problem was high at the $26^{\text {th }}$ week of the month.

Fig 3: The distribution of malaria outbreak by place, Gobera Kebele, Argoba district, Northeast Ethiopia, 2016. Shows the distribution of the problem among the kebeles in the district and it was high in Gobera kbele.

Additional file 1: Questionnaire, data collection tool. The questionnaire was developed by reviewing literatures for this study.

\section{Figures}




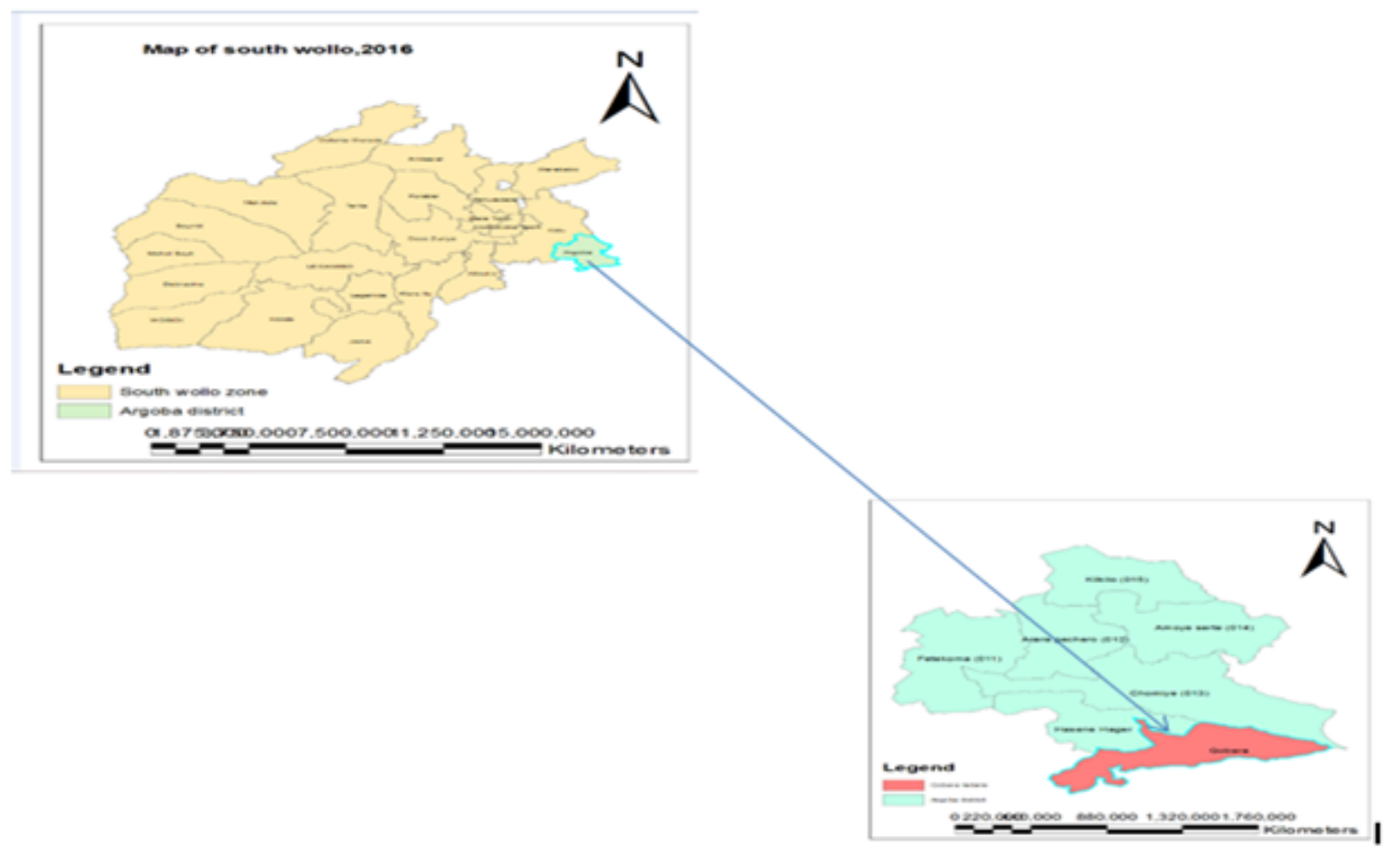

Figure 1

Map of Argoba district, 2016. Shows the location of Argoba district which is located at the East of the zone, south wollo Zone.

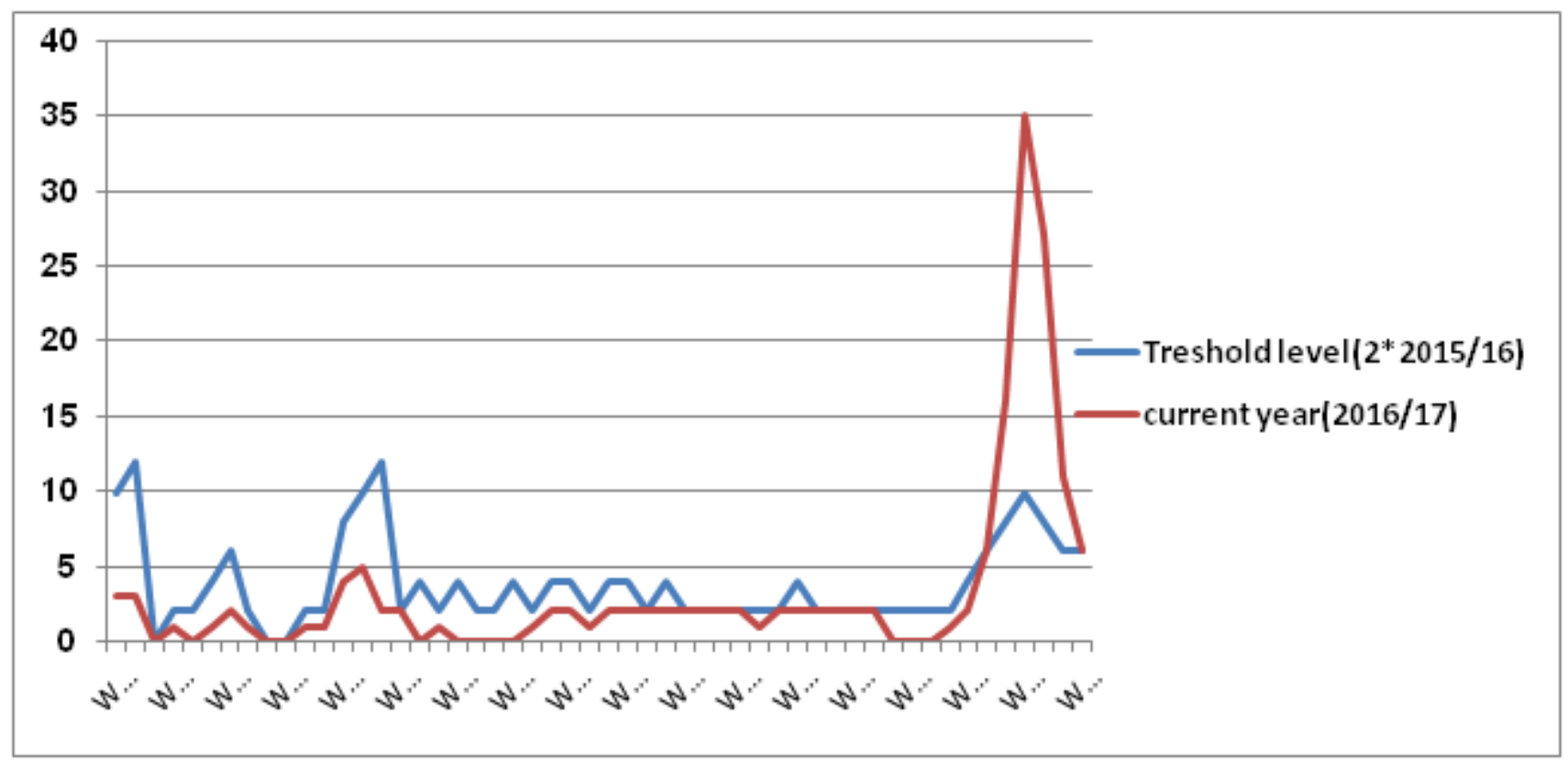

Figure 2

Malaria cases by weeks in Gobera, Argoba district, 2016. Illustrates distribution of malaria by time in the district. The magnitude of the problem was high at the 26th week of the month. 


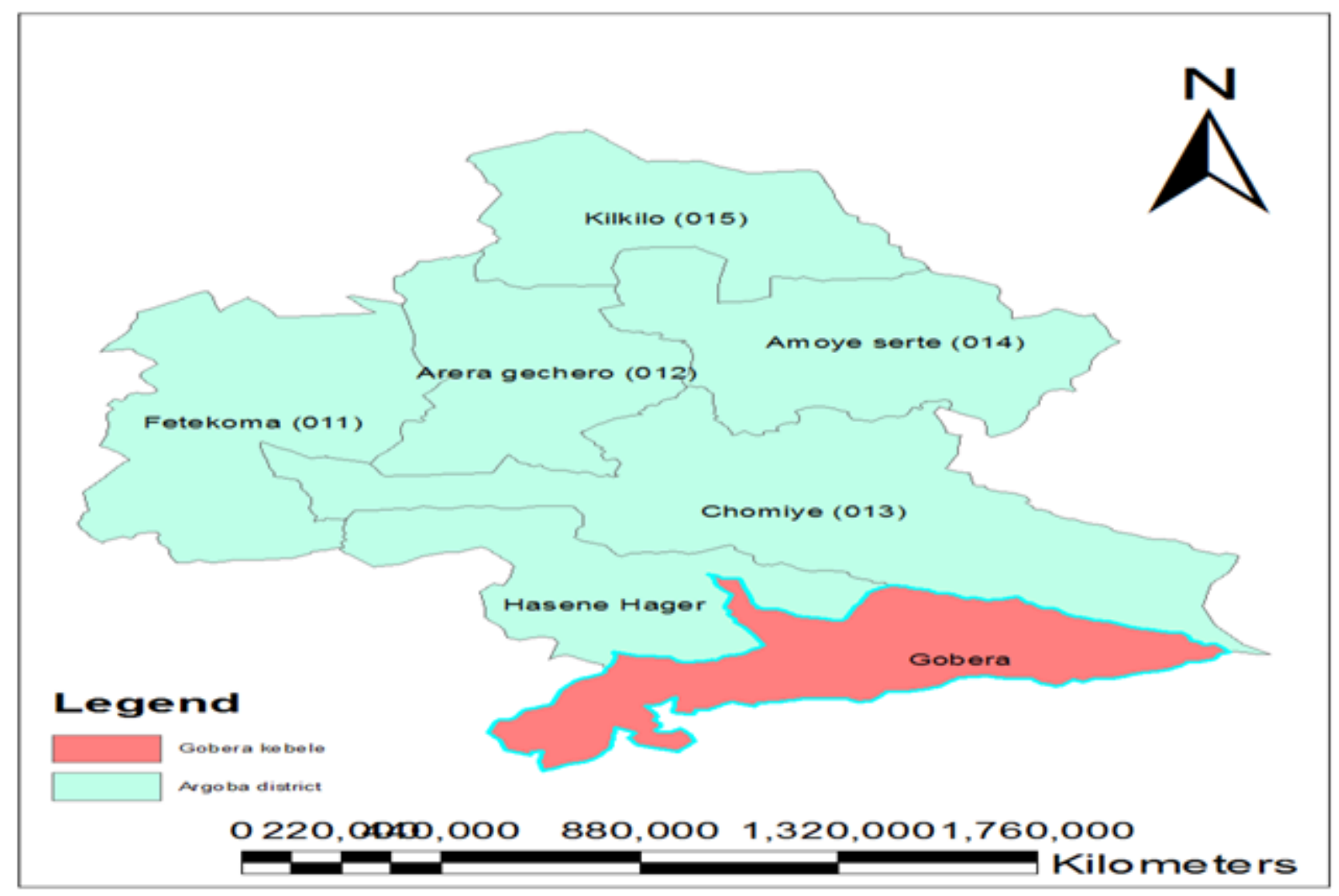

Figure 3

The distribution of malaria outbreak by place, Gobera Kebele, Argoba district, Northeast Ethiopia, 2016.

Shows the distribution of the problem among the kebeles in the district and it was high in Gobera kbele.

\section{Supplementary Files}

This is a list of supplementary files associated with this preprint. Click to download.

- Additionalfile1.pdf 